\title{
MOBILITY SPECTRUM ANALYSIS OF THE ELECTRICAL CONDUCTION
}

\author{
Z. Dzivba \\ Institute of Plysics, Polish Academy of Sciences \\ Al. Lotników 32/46, 02-668 Warszawa, Poland \\ (Received October 1, 1991)
}

\begin{abstract}
The electrical conductivity tensor components $\sigma_{x x}(H)$ and $\sigma_{x y}(H)$ have been transformed into mobility spectrum. The resolution of the mobility spectrum has been discussed. As an example the electrical conduction in HgTe and HgCdTe samples have been analyzed using resistivity tensor components, conductivity tensor components and using the mobility spectrum.
\end{abstract}

PACS numbers: 72.15.Eb, 72.20.My, 72.80.Ey

\section{Introduction}

The integral expressions describing the electrical conduction in a periodic system is usually derived from the transport theory in the effective mass approximation. The integration over the $k$ vector space was obtained by McClure in 1956 [1]. A commonly used form of integration in the energy space $E$ was derived by Kolodziejczak in 1961 [2]. However, the most useful approach to the analysis of the electrical conduction versus magnetic field was proposed in 1987 by Beck and Anderson [3]. There the integration runs over the mobility $\mu$. All above-mentioned methods express the electrical conduction as an integral of the spectrum of the electrical conductivity density $S(k), S(E)$ or $s(\mu)$. The electrical conductivity density $s(\mu)$ has been called by Beck and Anderson the mobility spectrum [3].

The electrical conductivity tensor components versus magnetic field $\sigma_{x x}(H)$ and $\sigma_{x y}(H)$ can be transformed into the mobility spectrum $s(\mu)$ [4]. This transformation is related to the inverse Laplace transform $[4,5]$. The mobility spectrum $s(\mu)$ is a new tool in the analysis of the experimental data, complementary to resistivity tensor or conductivity tensor components. On the other hand, the mobility spectrum theoretically calculated or experimentally determined is a new form of 
presentation of electrical carrier parameters, instead of the commonly used parameters: concentration $n$, average mobility $\mu$, and Hall factor $r$. The analysis of the mobility spectrum seems to be more fundamental than the analysis of the average carrier parameters: $n$ and $\mu$.

The numerical procedure of transformation of the electrical conductivity tensor components into the mobility spectrum described in Ref. [4] has been modified and is presented in this paper. The errors of the mobility spectrum are discussed. As an example, the mobility spectrum for HgTe sample has been analyzed.

\section{Progress in the analysis of the electrical conduction}

Properties of semiconductors are described by using models of physical phenomena, in the case of the transport phenomena using the model of the band structure and models of the scattering mechanisms (Fig. 1). Electrical conduction

(a)

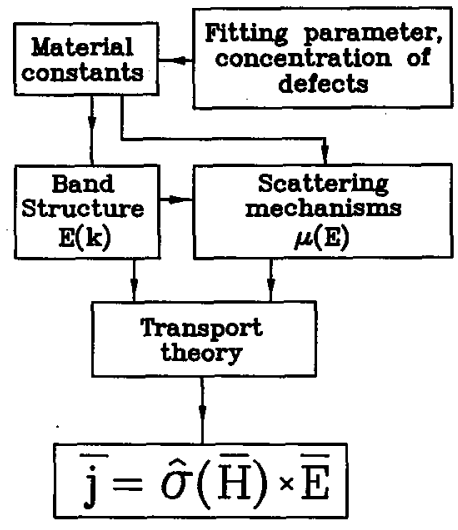

(b)

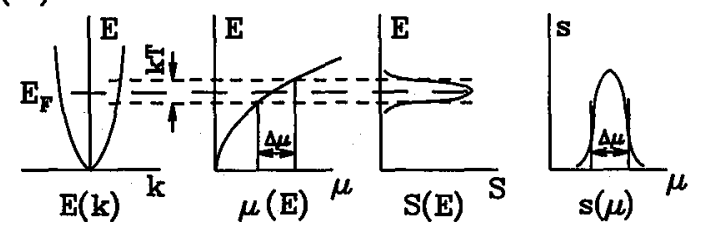

$$
\begin{aligned}
& \sigma_{z x(H)+i} \sigma_{x y}(H)=\int_{-\infty}^{\infty} \frac{S(E)(1+i \mu(E))}{1+(\mu(E) H)^{2}} d E \quad \text { Ref.[2] } \\
& \sigma_{z x}(\mathrm{H})+i \sigma_{z y}(\mathrm{H})=\int_{-\infty}^{\infty} \frac{\mathrm{s}(\mu)(1+i \mu)}{1+(\mu \mathrm{H})^{2}} \mathrm{~d} \mu \quad \text { Ref.[3] }
\end{aligned}
$$

Fig. 1. The algorithm of the transport theory and definition of the tensor conductivity $\widehat{\sigma}(H)=\sigma_{x x}(H)+\mathrm{i} \sigma_{x y}(H)(\mathrm{a})$, physical interpretation of the energy spectrum $S(E)$ and the mobility spectrum $s(\mu), S(E)$ and $s(\mu)$ are density of the electrical conductivity (b).

versus magnetic field is a powerful tool in the analysis of the transport theory. The experimental data of the electrical conduction can be expressed by the resistivity $\hat{\rho}(H)$ or conductivity $\widehat{\sigma}(H)$ tensor components. Using the familiar theoretical expressions valid in the non-quantum regime [2], the $\widehat{\sigma}(H)$ can be transformed into the mobility spectrum $[3,4]$. Then, we have a new tool in the analysis of the transport theory, the mobility spectrum.

In order to describe the electrical conduction the parameters derived from the transport theory are used. Parameterizing the electrical conduction can be realized in different levels of the theory. Commonly used parameters are the concentration and the mobility of the electric carriers. They are calculated from the Hall coefficient and conductivity using the familiar expressions valid in the case of 
the conduction by one group of carriers $[2,3]$ or in the case of mixed conduction from the analysis of the magnetic field dependence of the electrical conductivity tensor components $\sigma_{x x}(H), \sigma_{x y}(H)[6,7]$. However, more fundamental description of the electrical conduction is made by the mobility spectrum $s(\mu)$. It is seen in Fig. 1 that the shape of the mobility spectrum peak depends on the band structure $E(k)$ and on the scattering mechanism $\mu(E)$ in the range of the energy $k T$ close to the Fermi energy $E_{\mathrm{F}}$. In contrary to the average carrier parameters the experimentally determined mobility spectrum provides a suggestive representation of the electrical conduction that is easily interpreted in terms of the band structure and scattering processes.

On the other hand, the average carrier parameters can not very well describe the magnetic field dependence of the electrical conduction [4]. It follows from the integral expressions describing $\widehat{\sigma}(H)$ (see Fig. 1). In order to describe $\widehat{\sigma}(H)$ precisely the mobility spectrum $s(\mu)$ is necessary [4].

For example, the electrical conduction for $\mathrm{HgCdTe}$ sample at the temperature $0.4 \mathrm{~K}$ analyzed by Tsidilkovskii et al. $[8,9]$ is shown in Fig. 2 in forms of the resistivity tensor components, conductivity tensor components, and in forms of the mobility spectrum of partial conductions for electrons and holes.

(a)

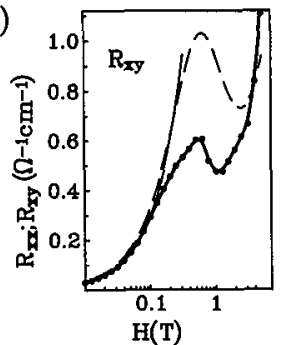

(b)

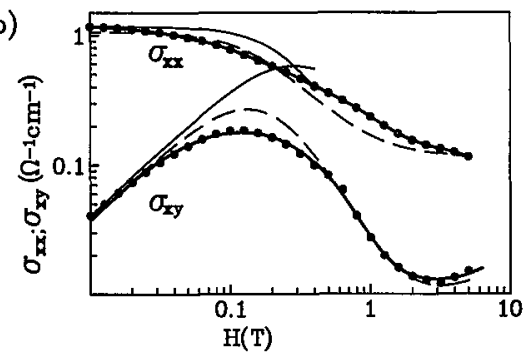

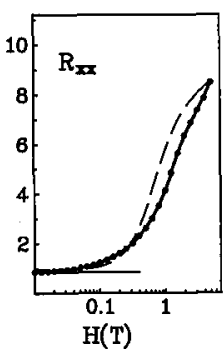

(c)

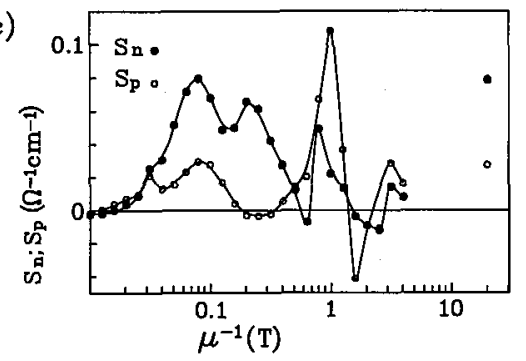

Fig. 2. The electrical conduction for $\mathrm{HgCdTe}$ sample at the temperature $0.4 \mathrm{~K}[8,9]$ are shown in the forms: (a) - resistivity tensor components, (b) - conductivity tensor components, (c) - mobility spectrum for electrons and holes.

The resistivity tensor components $\rho_{x x}(H)$ and $\rho_{x y}(H)$ have been analyzed by Tsidilkovskii et al. $[8,9]$ assuming that the whole transport is realized by conduction band electrons. The parameters for electrons $[8,9]$ are shown in Table I. 
With the use of these parameters the resistivity and conductivity tensor components in low magnetic fields have been calculated. The results are shown in Figs. 2a and $2 b$ by thin solid lines. Good agreement between calculated curves and experimental data in low magnetic fields have been obtained. The resistivity tensor components in high magnetic fields were described assuming magnetic localization of the conduction band electrons. The critical magnetic fields were estimated from characteristic points on $\rho_{x x}(H)$ and $\rho_{x y}(H)$ curves $[8,9]$.

\section{TABLE I}

The conduction and mobility of electrons calculated in Ref. $[5,6]$ for the single band model, and the conductions and mobilities of high and low mobility electrons and holes calculated for the multiband model.

\begin{tabular}{l|l|r|r|r|l}
\hline \hline & $\begin{array}{c}S \\
(\Omega \mathrm{cm})^{-1}\end{array}$ & $\begin{array}{c}\mu \\
\mathrm{m}^{2} / \mathrm{Vs}\end{array}$ & $\begin{array}{c}S \\
(\Omega \mathrm{cm})^{-1}\end{array}$ & $\begin{array}{c}\mu \\
\mathrm{m}^{2} / \mathrm{Vs}\end{array}$ & \\
\hline$n$ & 1.1 & 35000 & & & Ref. $[8,9]$ \\
$n$ & 0.7 & 63000 & 0.08 & 500 & 7 parameter fit \\
$p$ & 0.24 & 25000 & 0.04 & 500 & \\
$n$ & 0.64 & 110000 & 0.16 & 5800 & from mobility spectrum \\
$p$ & 0.16 & 200000 & 0.22 & 15000 &
\end{tabular}

The conductivity tensor components $\sigma_{x x}$ and $\sigma_{x y}$ calculated from $\rho_{x x}$ and $\rho_{x y}[6]$ are shown in Fig. 2b. Assuming that the quantum effects can be negligible, the average carrier parameters have been calculated as 7 parameter fit to $\sigma_{x x}(H)$ and $\sigma_{x y}(H)$ experimental data. In the fitting the integral expressions [2] have been approximated by algebraic expressions [7] putting all scattering coefficients being equal to one. The resulting parameters are shown in Table I. The resistivity and conductivity tensor components calculated with the use of the fitting parameters are shown by broken lines in Figs. $2 \mathrm{a}$ and $2 \mathrm{~b}$. There is a quite good agreement between the calculated curves and experimental data for $\rho_{x x}(H), \sigma_{x x}(H)$, and $\sigma_{x y}(H)$, and not so good for $\rho_{x y}(H)$. Disagreement between the calculated curves and experimental data follows from the approximations in which we have used all scattering coefficients being equal to one $[6,7]$, and we have neglected the quantum effects.

The result of the transformation of $\sigma_{x x}(H)$ and $\sigma_{x y}(H)$ into the mobility spectrum $s^{n}(\mu)$, and $s^{p}(\mu)$ is shown in Fig. 2c. Assuming that the quantum effects are negligible, the mobility spectrum presents the full physical information which can be obtained from the analysis of the $\sigma_{x x}(H)$ and $\sigma_{x y}(H)$ experimental data. The quality of the phenomenological description of the magnetic field dependence of the electrical conduction using the mobility spectrum is shown by thick solid lines in Figs. 2a and 2b. With all excellent descriptions of the experimental data, the mobility spectrum shown in Fig. $2 \mathrm{c}$ presents unphysical results. Negative partial conductions were obtained in high magnetic field. It follows from the quantum effects which can not be neglected particularly in high magnetic fields. However, assuming that the quantum effects can be neglected the average parameters $[4,7]$ 
for high and low mobility groups of electrons and holes have been calculated and the results are shown in Table I.

The above example shows the possibility following from the analysis of the experimental data in the form of resistivity tensor components, conductivity tensor components, and in the form of the mobility spectrum.

Note that the resistivity tensor components are very useful in the analysis of the electrical conduction in the single band structure model, whereas the conductivity tensor components are very useful in the case of the multiband structure model. However, the mobility spectrum shows the properties which are not seen on the curves of the conductivity or resistivity tensor components. In the analysis of the electrical conduction the mobility spectrum is a complementary form to the resistivity and conductivity tensor components.

It is clear that the parameters describing the electrical conduction depend on the model and on the approximations used in the analysis (Table I). The differences between the carrier parameters follow from different models used in the analysis of the electrical conduction as well as from the approximations. It must be pointed out that the average mobilities calculated from the mobility spectrum correspond to the Hall mobility [7]. The mobilities obtained in the fitting procedure are undefined and can differ by the scattering coefficient from the mobilities obtained from the mobility spectrum. Both groups of parameters contain an error due to neglecting the quantum effects observed in high magnetic field. The parameters obtained with the assumption of the single band and magnetic localization of the conduction band electrons $[8,9]$ can not be compared with the parameters estimated here while neglecting all quantum effects in the multiband energy structure.

\section{Conductivity tensor components}

In the transformation of the conductivity tensor components $\sigma_{x x}(H)$ and $\sigma_{x y}(H)$ into the mobility spectrum $s^{n}(\mu), s^{p}(\mu)$ the integral expressions [3] have been approximated by the sum expressions [4]:

$$
\begin{aligned}
& \sigma_{x x}(H)=\int_{0}^{\infty} \frac{\left[s^{n}(\mu)+s^{p}(\mu)\right] \mathrm{d} \mu}{1+\mu^{2} H^{2}}=\sum_{i} \frac{\left[s_{i}^{n}(\mu)+s_{i}^{p}(\mu)\right] \Delta \mu_{i}}{1+\mu_{i}^{2} H^{2}} \\
& \frac{\sigma_{x y}(H)}{H}=\int_{0}^{\infty} \frac{\left[s^{n}(\mu)-s^{p}(\mu)\right] \mu \mathrm{d} \mu}{1+\mu^{2} H^{2}}=\sum_{i} \frac{\left[s^{n}\left(\mu_{i}\right)-s^{p}\left(\mu_{i}\right)\right] \mu_{i} \Delta \mu_{i}}{1+\mu_{i}^{2} H^{2}},
\end{aligned}
$$

where $\mu_{i}$ covers a whole range of the mobility with the step $\Delta \mu_{i}$. The sets $\left(s^{n}\left(\mu_{i}\right) \Delta \mu_{i}, \mu_{i}\right),\left(s^{p}\left(\mu_{i}\right) \Delta \mu_{i}, \mu_{i}\right)$ correspond to the mobility spectrum $s^{n}(\mu), s^{p}(\mu)$.

To simplify the notation, it is useful to introduce effective amplitudes $A_{i}^{x x}$ and $A_{i}^{x y}$ :

$$
\begin{aligned}
& A_{i}^{x x}=\left[s^{n}\left(\mu_{i}\right)+s^{p}\left(\mu_{i}\right)\right] \Delta \mu_{i}=S_{i}^{n}+S_{i}^{p}, \\
& A_{i}^{x y}=\left[s^{n}\left(\mu_{i}\right)-s^{p}\left(\mu_{i}\right)\right] \Delta \mu_{i}=S_{i}^{n}-S_{i}^{p},
\end{aligned}
$$

where $S_{i}^{n}$ and $S_{i}^{p}$ are partial conductions of electrons and holes, respectively. 
According to Ref. [4] the set of the experimental data $\left[\sigma_{x x}\left(H_{j}\right), \sigma_{x y}\left(H_{j}\right), H_{j}\right]$ can be transformed into the set $\left[A_{i}^{x x}, A_{i}^{x y}, \mu_{i}\right]$, and then the set $\left[A_{i}^{x x}, A_{i}^{x y}, \mu_{i}\right]$ can be easily transformed into $s(\mu)$ [4]. The mobilities $\mu_{i}$ in Eqs. (1) and the magnetic fields $H_{j}$ in the set $\left[\sigma_{x x}\left(H_{j}\right), \sigma_{x y}\left(H_{j}\right), H_{j}\right]$ are given by [4]:

$$
\begin{aligned}
& \mu_{i}=10^{\left(i_{\max }-i\right) / d} \\
& H_{j}=10^{\left(j+j_{\min }\right) / d}
\end{aligned}
$$

where $i, j$, and $d$ are the integer numbers, $d$ determines density of points on a $\operatorname{logarithmic} \mu$ and $H$-axis, $i_{\max }=d \log \left(\mu_{\max }\right)+1, j_{\min }=d \log \left(H_{\min }\right)-1$, $\mu_{\max } H_{\min }=1$.

Substituting Eqs. (2) into Eqs. (1) one gets:

$$
\begin{aligned}
& \sigma_{x x}\left(H_{j}\right)=\sum_{i} A_{i}^{x x} m_{i, j} \\
& \sigma_{x y}\left(H_{j}\right) / H_{j}=\sum_{i} A_{i}^{x y} \mu_{i} m_{i, j},
\end{aligned}
$$

where

$$
\left[m_{i, j}\right]=\left|\begin{array}{lllll}
a_{0} & a_{1} & a_{2} & \ldots & a_{n} \\
a_{-1} & a_{0} & a_{1} & \ldots & a_{n-1} \\
a_{-2} & a_{-1} & a_{0} & \ldots & a_{n-2} \\
\ldots \ldots \ldots \ldots \ldots \ldots \ldots \ldots \ldots \ldots \ldots & \ldots \ldots \ldots \\
a_{-n} & a_{-n+1} & a_{-n+2} & \ldots & a_{0}
\end{array}\right|
$$

with $2 n+1$ elements $a_{k}$ :

$$
a_{k}=\frac{1}{1+10^{2 k / d}},
$$

where $k=j-i$.

The tensor components $\sigma_{x x}\left(H_{j}\right)$ and $\sigma_{x y}\left(H_{j}\right)$ may be calculated from Eqs. (3) easier than using Eqs. (1).

\section{Mobility spectrum}

The electrical conduction is transformed into the mobility spectrum using an iterative procedure, similar to that described in Ref. [4].

In the iterations the subsequent trial functions for $\sigma_{x x},\left(A_{i, k}^{x x}, \mu_{i}\right)$ are the set of amplitudes $A_{i}^{x x}$ and mobilities $\mu_{i}$. The first trial function $\left(A_{i, 1}^{x x}, \mu_{i}\right)$ can be calculated using the envelope of the mobility spectrum obtained by the method described in Ref. [3] or a test function described in Ref. [4]. Also, the fitting parameters can be used in the estimation of the first trial function. Further the trial functions $\left(A_{i, k}^{x x}, \mu_{i}\right)$ are estimated in the iteration procedure, until the calculated conduction tensor component attains good agreement with the experimental data. The algorithm of the iterations is shown in Fig. 3. The basic iteration procedure 
Simple procedure

Extended procedure
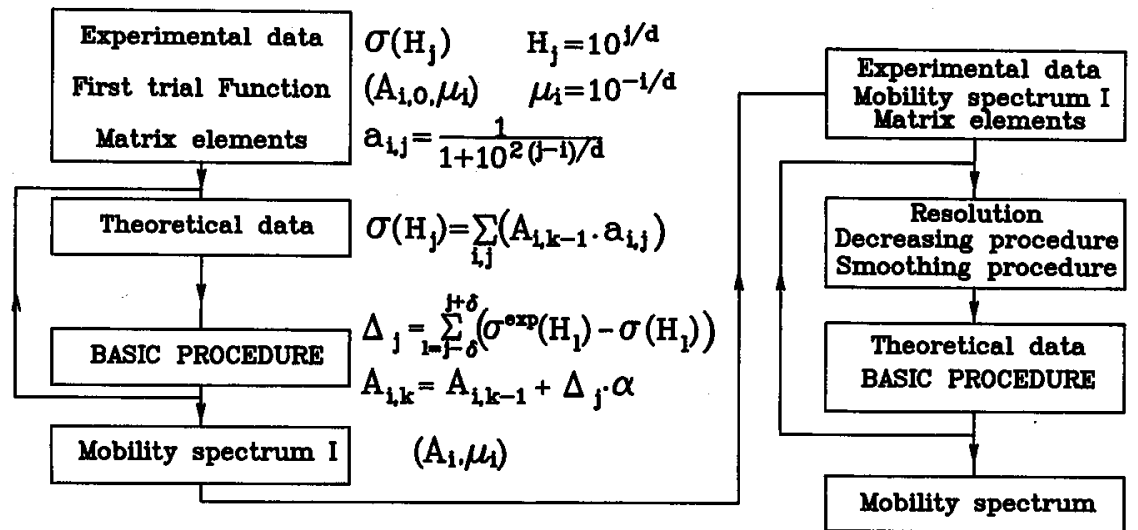

Fig. 3. The algorithm of the numerical transformation of $\sigma(H)$ into $s(\mu)$. The simple procedure is used in the first step and the extended procedure in the final step of the transformation.

is given by the following expressions:

$$
\begin{aligned}
& \Delta_{i}=\sum_{j=i-\delta}^{i+\delta}\left[\sigma_{x x}^{\exp }\left(H_{j}\right)-\sigma_{x x}\left(H_{j}\right)\right], \\
& A_{i, k}^{x x}=A_{i, k-1}^{x x}+\Delta_{i} \alpha .
\end{aligned}
$$

The conductions $A_{i, k}^{x x}$ are obtained after $k$ iterations. The quantities $\delta$ and $\alpha$ are adjustment coefficients, $\sigma_{x x}^{\exp }\left(H_{j}\right)$ are experimental data and $\sigma_{x x}\left(H_{j}\right)$ are calculated from Eq. (3a). The trial functions and the basic iteration procedure for $\sigma_{x y}$ component are defined similarly to that shown above for $\sigma_{x x}$.

For $\delta=0$, the basic procedure $(4 \mathrm{a}, \mathrm{b})$ is the same as the basic procedure described in Ref. [4], and it transforms randomly distributed experimental data $\left[\sigma_{x x}\left(H_{j}\right), \sigma_{x y}\left(H_{j}\right), H_{j}\right]$ into randomly distributed amplitudes of the set $\left[A_{i}^{x x}, A_{i}^{x y}, \mu_{i}\right]$. In order to decrease this random distribution of the amplitudes $A^{x x}$ and $A^{x y}$ we introduced $\delta>0$. We calculated $\Delta_{i}$ for magnetic fields in the range $H_{i-\delta}-H_{i+\delta}$ where $H_{i}$ satisfies the following relation:

$$
H_{i}=1 / \mu_{i} \text {. }
$$

\section{Properties of the mobility spectrum}

In order to obtain the mobility spectrum $s(\mu),\left[s^{\mathrm{n}}(\mu), s^{\mathrm{P}}(\mu)\right]$ we ought to transform a continuous function $\widehat{\sigma}^{\exp }(H)=\sigma_{x x}^{\exp }(H)+\mathrm{i} \sigma_{x y}^{\exp }(H)$, covering magnetic field range from $H=0$ to $H=\infty$ and given with an infinite accuracy, into 
a continuous function $s(\mu)$. However, the iteration method works while using a finite number of experimental data which have a finite accuracy and which are in a limited magnetic field range. Because the transformation of the conductivity tensor $\hat{\sigma}^{\exp }(H)$ into the mobility spectrum $s(\mu)$ is very unstable $[4,5]$, we can obtain a family of curves $s(\mu), F(s(\mu))$, or a family of sets $\left[A_{i}^{x x}, A_{i}^{x y}, \mu_{i}\right], F\left(\left[A_{i}^{x x}, A_{i}^{x y}, \mu_{i}\right]\right)$, which describe the experimental data $\widehat{\sigma}^{\exp }(H)$ within the experimental error.

The differences between the sets $\left[A_{i}^{x x}, A_{i}^{x y}, \mu_{i}\right]$ of the family $F\left(\left[A_{i}^{x x}, A_{i}^{x y}, \mu_{i}\right]\right)$ are due to the following property. Two amplitudes $A_{j}^{x x}, A_{k}^{x x}$ in the set $\left(A_{i}^{x x}, \mu_{i}\right)$ describing the component $\sigma_{x x}(H)$ (Eq. 1a) may be replaced by the following amplitudes:

$$
A_{j}^{x x}=A_{j}^{x x}+A^{\prime} \text { and } A_{k}^{, x x}=A_{k}^{x x}-A^{\prime}
$$

and for all the rest amplitudes:

$$
A_{i}^{x x}=A_{i}^{x x} \text { for } i \neq j, k \text {. }
$$

From the new set $\left(A_{i}^{x x}, \mu_{i}\right)$ we can calculate the electrical conduction $\sigma_{x x}^{*}(H)$, which satisfies the relation:

$$
\sigma_{x x}(H)-\sigma_{x x}^{\prime}(H) \leq A^{\prime} \frac{\mu_{k}-\mu_{j}}{\mu_{k}+\mu_{j}}
$$

in the whole range of the magnetic field.

If $\sigma_{x x}^{\prime}(H)$ describes the experimental data within the error, the new set $\left(A_{i}^{x x}, \mu_{i}\right)$ belongs to the family $F\left(\left[A_{i}^{x x}, A_{i}^{x y}, \mu_{i}\right]\right)$. The difference between the sets $\left[A_{i}^{x x}, \mu_{i}\right],\left[A_{i}^{x x}, \mu_{i}\right]$ can be treated here as a result of a "loan" of $A$, from the amplitude $A_{k}^{x x}$ to the amplitude $A_{j}^{x x}$.

Using the "loan" technique, we can calculate the resolution of the mobility spectrum. The resolution used in this paper in the analysis of the set $\left[A_{i}^{x x}, A_{i}^{x y}, \mu_{i}\right]$ can be treated similarly to the resolution used in the analysis of optical spectra.

A possible definition of the resolution is presented below. "Left hand side resolution" of the mobility spectrum $\left(A_{i}^{x x}, \mu_{i}\right)$ at the point $i=z, \mu_{z}$, is a maximal range of mobilities $\Delta \bar{\mu}_{z^{-}}$:

$$
\Delta \bar{\mu}_{z-}=\mu_{z-\kappa}-\mu_{z},
$$

the number $\kappa$ denotes the range of mobilities, in which all amplitudes $A_{i}^{x x}$ can be replaced by one effective amplitude $A_{z}^{x x}$ at $\mu_{z}$. This way the set $\left(A_{i}^{x x}, \mu_{i}\right)$ is transformed into a set $\left(A_{i}^{x x}, \mu_{i}\right)$ :

$$
\begin{aligned}
& A_{z}^{x x}=\sum_{j=z-\kappa}^{z} A_{j}^{x x}, \\
& A_{j}^{x x}=0, \quad \text { for } z-\kappa<j<z \\
& A_{j}^{x x}=A_{j}^{x x}, \quad \text { for } j<z-\kappa \text { and } j>z
\end{aligned}
$$

and the new fit with the use $\left(A_{i}^{x x}, \mu_{i}\right)$ is within the experimental error. 
In a similar way we can define the "right hand side resolution" $\Delta \bar{\mu}_{z+}$. Using above definitions for the set $\left(A_{i}^{x x}, \mu_{i}\right)$ and similar for the set $\left(A_{i}^{x y}, \mu_{i}\right)$ we can calculate the resolution $\Delta \bar{\mu}_{i}=\Delta \bar{\mu}_{i-}+\Delta \bar{\mu}_{i+}$ at each point $\mu_{i}$ for every set $\left[A_{i}^{x x}, A_{i}^{x y}, \mu_{i}\right]$ of the family $F\left(\left[A_{i}^{x x}, A_{i}^{x y}, \mu_{i}\right]\right)$. The resolution can be determined by the range $\Delta \bar{\mu}_{i}$ or by the number $\kappa$.

The resolutions for $A_{i}^{x x}$ and similarly defined resolutions for $A_{i}^{x y}$ describe local properties of the set $\left[A_{i}^{x x}, A_{i}^{x y}, \mu_{i}\right]$. It is clear that the structure of the mobility spectrum within the range $\Delta \bar{\mu}_{i}$ can be different in different sets $\left[A_{i}^{x x}, A_{i}^{x y}, \mu_{i}\right]$ and has no physical meaning.

\section{Example}

The conductivity tensor components for a HgTe sample at the temperature $30 \mathrm{~K}$ versus magnetic field in the range from 0.01 to $30 \mathrm{~T}$ are shown in Fig. 4.

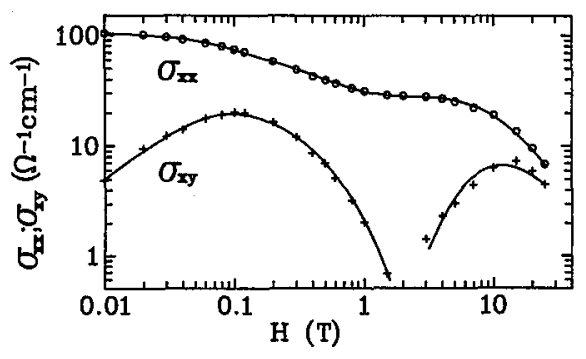

Fig. 4. The experimental data of the tensor components $\sigma_{x x}(000)$ and $\sigma_{x y}(\mathrm{xxxx})$ for $\mathrm{HgTe}$ at the temperature $30 \mathrm{~K}$. Curves calculated with the use of the mobility spectra were obtained from the first trial function using fitting parameters for $\sigma_{x y}$ component.

From the analysis of the conductivity tensor components similar to that used in Ref. [6] the conductions and mobilities of electrons and holes have been obtained: $\sigma_{n}, \mu_{n}, \sigma_{p}, \mu_{p}$. Two groups of parameters have been estimated. From $\sigma_{x x}$ component: $\sigma_{n}=70(\Omega \mathrm{cm})^{-1}, \mu_{n}=50000 \mathrm{~cm}^{2} / \mathrm{Vs}, \sigma_{p}=30(\Omega \mathrm{cm})^{-1}, \mu_{p}=$ $1000 \mathrm{~cm}^{2} / \mathrm{Vs}$ and from $\sigma_{x y}$ component: $\sigma_{n}=40(\Omega \mathrm{cm})^{-1}, \mu_{n}=10000 \mathrm{~cm}^{2} / \mathrm{Vs}$, $\sigma_{p}=14(\Omega \mathrm{cm})^{-1}, \mu_{p}=1000 \mathrm{~cm}^{2} / V \mathrm{~s}$. The four parameters obtained from $\sigma_{x x}$ have been used in the calculation of the first trial function for $\sigma_{x x}$ and the first trial function for $\sigma_{x y}$ as follows:

$$
A_{i}^{x x}=0 \text { and } A_{i}^{x y}=0
$$

for all $i$ except these ones for which $\mu_{i}$ is closest to $\mu_{n}$ or $\mu_{p}$. There:

$$
\begin{aligned}
& A_{i}^{x x}=\sigma_{n}+\sigma_{p}, \\
& A_{i}^{x y}=\sigma_{n}-\sigma_{p} .
\end{aligned}
$$


Using Eqs. (2), (3)and (4) with the parameters: $d=10, \alpha=0.01$, and $\delta=5$ the experimental data $\hat{\sigma}^{\exp }\left(H_{j}\right)$ have been transformed into the set $\left[A_{i}^{x x}, A_{i}^{x y}, \mu_{i}\right]$. In Fig. 5 the set $\left(A_{i}^{x x}, \mu_{i}\right)$ obtained after 50 iterations is marked with crosses.

Another set $\left[A_{i}^{x x}, A_{i}^{x y}, \mu_{i}\right]$ has been obtained starting with the four parameters $\sigma_{n}, \mu_{n}, \sigma_{p}, \mu_{p}$ estimated from $\sigma_{x y}$. The resulting set $\left(A_{i}^{x x}, \mu_{i}\right)$ is marked in Fig. 5 with circles. In each set two components $\left(A_{i}^{x x}, \mu_{i}\right)$ with the parameters close to the starting parameters $\sigma_{n}, \mu_{n}, \sigma_{p}, \mu_{p}$ are observed. Because both sets $\left(A_{i}^{x x}, \mu_{i}\right)$ describe the electrical conduction with similar accuracy, only the fit given by the mobility spectrum obtained with the starting parameters from $\sigma_{x x}$ is shown by solid lines in Fig. 4.

The resolutions $\Delta \bar{\mu}_{i}$ for the set $\left[A_{i}^{x x}, A_{i}^{x y}, \mu_{i}\right]$ have been calculated from Eqs. (6). In Fig. 5 the resolutions for the mobility spectrum $\left(A_{i}^{x x}, \mu_{i}\right)$ marked with circles are shown by horizontal lines. The resolutions for the investigated mobility spectrum overlap strongly.

In order to obtain more precise transformation of the electrical conduction into mobility spectrum, the basic iteration procedure is supported with a decreasing procedure and a smoothing procedure. The decreasing procedure for amplitude $A_{i}^{u v}(u v=x x, x y)$ has a simple form:

$$
A_{i}^{u v}=k A_{i}^{u v}, \quad \text { where } k<1 .
$$

The smoothing procedure, similar to that used in Ref. [4], transforms the amplitude $A_{i}^{u v}$ into $A_{i}^{u v}$ as follows:

$$
A_{i}^{u v}=0.5\left(A_{i}^{u v}+\bar{A}_{i}\right),
$$

where $\bar{A}_{i}$ is calculated from a linear equation:

$$
\frac{\bar{A}_{i}-A_{i-1}^{u v}}{\mu_{i}-\mu_{i-1}}=\frac{A_{i+1}^{u v}-\bar{A}_{i}}{\mu_{i+1}-\mu_{i}}
$$

The algorithm of the extended iteration procedure contäining basic, decreasing and smoothing procedures described by Eqs. $(4,8,9)$ is shown in Fig. 3. In each iteration the decreasing procedure, the smoothing procedure, and the basic procedure are repeated for all points $\mu_{i}$. At a point $\mu_{i}$ the decreasing procedure is used for all amplitudes $A_{j}^{u v}$ in the range $\Delta \bar{\mu}_{i}$ excluding the amplitude $A_{i}^{u v}$. Then, in each iteration all amplitudes $A_{i}^{u v}$ are decreased proportionally to the number of horizontal lines covering $A_{i}^{u v}$. Similarly, the smoothing procedure is repeated for all ranges $\Delta \bar{\mu}_{i}$. However, the minimal range $\Delta \bar{\mu}_{i}$ in the smoothing procedure is given by three points: $i-1, i, i+1$, even in the case $\kappa=0$.

Starting with two mobility spectra marked in Fig. 5 with circles and crosses, the final mobility spectra have been calculated using the extended iteration procedure. The mobility spectra obtained after 10 iterations are shown in Fig. 6. Both spectra are similar. The resolutions for only one mobility spectrum (circles) are shown in Fig. 6 because for the both sets the resolutions are also similar. It is seen that the resolutions for the main groups of amplitudes in the range of mobilities from $20000 \mathrm{~cm}^{2} / \mathrm{V}$ s to $300000 \mathrm{~cm}^{2} / \mathrm{V} \mathrm{s}$, and from $200 \mathrm{~cm}^{2} / \mathrm{V} \mathrm{s}$ to $2000 \mathrm{~cm}^{2} / \mathrm{V} \mathrm{s}$ 

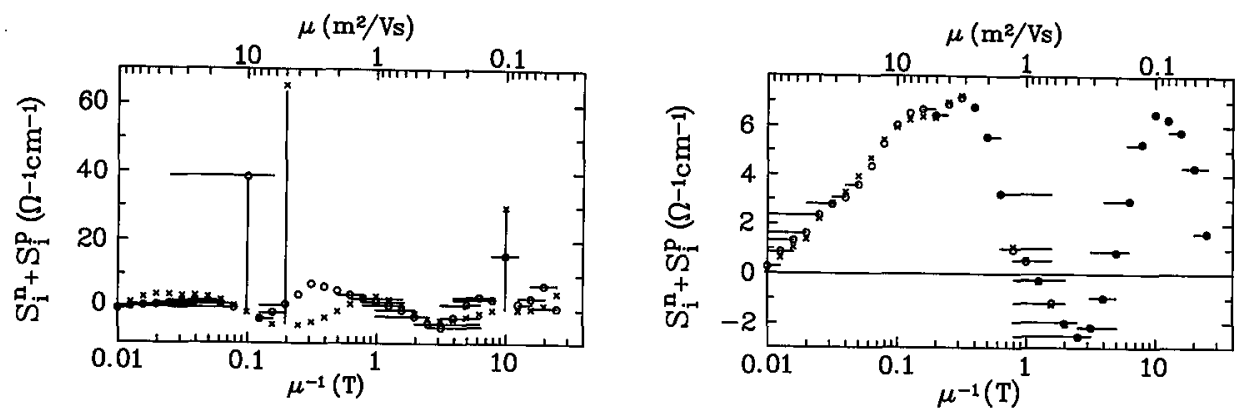

Fig. 5. The mobility spectra for $\sigma_{x x}$ component obtained by using a simple version of the iteration procedure. The mobility spectrum ( $x \times x x$ ) was obtained by using the first trial function estimated from the fitting parameters for $\sigma_{x x}$. The mobility spectrum (oooo) and spectral resolutions (__ was obtained by using the first trial function estimated from the fitting parameters for $\sigma_{x y}$. The common scale for $H$ and $\mu^{-1}$ is useful in the comparison of the properties of the $\sigma(H)$ and mobility spectrum.

Fig. 6. The mobility spectra obtained from the extended procedure using the first trial functions obtained from the simple iteration procedure shown in Fig. 3. The circles (oooo) and crosses ( $x \times x x)$ correspond to circles and crosses in Fig. 3.

correspond to $\kappa \leq 1$. Only the tails of the main groups of amplitudes are characterized by $\kappa>2$.

It is interesting to compare the mobility spectra obtained with the use of the simple and extended iteration procedures. The mobility spectra marked in Fig. 5 and Fig. 6 with circles are shown in Fig. 7 by open and full circles. The loan effect is seen in the region close to the amplitudes $A=38(\Omega \mathrm{cm})^{-1}$ and $A=16(\Omega \mathrm{cm})^{-1}$.

Using the method described above we have selected from the family $F\left[\left(A_{i}^{x x}, A_{i}^{x y}, \mu_{i}\right)\right]$ a class of solutions $\left(A_{i}^{x x}, A_{i}^{x y}, \mu_{i}\right)$, which can not be modified using the "loan" technique defined by Eqs. (6). However, these solutions can be still modified using a "feeble loan" technique, in which the set $\left(A_{i}^{x x}, A_{i}^{x y}, \mu_{i}\right)$ will be modified using for loan procedure an amplitude $A$ ' chosen in such a way that a new set $\left(A_{i}^{x x}, A_{i}^{x y}, \mu_{i}\right)$ describe the experimental data and is still characterized by resolutions corresponding to $\kappa<1$.

In order to interpret the structure seen in the range of mobilities from $20000 \mathrm{~cm}^{2} / \mathrm{V}$ s to $300000 \mathrm{~cm}^{2} / \mathrm{V} \mathrm{s}$, the electron $S_{i}^{n}$ and hole $S_{i}^{p}$ contributions have been calculated from the amplitudes $A_{i}^{x x}$ and $A_{i}^{x y}$ as follows:

$$
\begin{aligned}
& S_{i}^{n}=0.5\left(A_{i}^{x x}+A_{i}^{x y}\right), \\
& S_{i}^{p}=0.5\left(A_{i}^{x x}-A_{i}^{x y}\right) .
\end{aligned}
$$

The results are shown in Fig. 8 , where $S_{i}^{n}$ are marked with crosses and $S_{i}^{p}$ with circles. The maxima of electron contribution are shifted with respect to the maxima of hole contribution. Note the negative conductions of electrons and holes in the range from $2000 \mathrm{~cm}^{2} / \mathrm{V} \mathrm{s}$ to $10000 \mathrm{~cm}^{2} / \mathrm{V} \mathrm{s}$ and for holes in the range from 

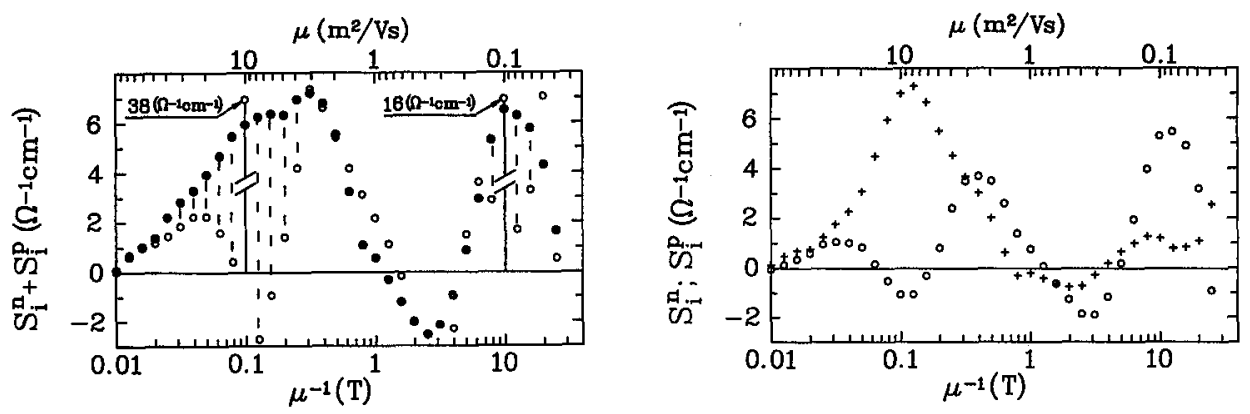

Fig. 7. A comparison of the mobility spectra obtained from the simple (oooo) and

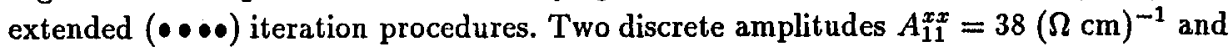
$A_{31}^{x x}=16(\Omega \mathrm{cm})^{-1}$ are due to the loans shown by broken lines.

Fig. 8. The mobility spectra of the electron $(++++)$ and hole (0000) contribution to the electrical conduction.

$50000 \mathrm{~cm}^{2} / \mathrm{V}$ s to $170000 \mathrm{~cm}^{2} / \mathrm{V}$ s. Completing the extended procedure with the conditions:

$$
\begin{aligned}
& S_{i}^{n} \geq 0 \\
& S_{i}^{p} \geq 0,
\end{aligned}
$$

we can force the electron and hole contributions to be positive. However, keeping the fit within the experimental error we can do it only for holes in the range of mobilities from $50000 \mathrm{~cm}^{2} / \mathrm{V}$ s to $170000 \mathrm{~cm}^{2} / \mathrm{V} \mathrm{s}$. It means that the negative conductions of holes in the higher mobility range are an artifact of errors in estimations of $A_{i}^{x x}$ and $A_{i}^{x y}$, and can be corrected in further analysis. The negative conductions in the range of low mobility can be interpreted as following from systematic experimental errors of $\sigma_{x x}$ and $\sigma_{x y}$ in the range of high magnetic fields, or as quantum effects, which lead to the magnetic field dependence of the concentrations and mobilities. This problem should be considered separately.

\section{Conclusions}

The electrical conduction versus magnetic field has been transformed into the mobility spectrum using the extended iteration procedure. In order to avoid the effect of the random dispersion of the experimental data on the resulting mobility spectrum, the corrections have been calculated from average deviations. In order to reduce the effect of choice of the first trial function on the resulting spectra, the resolution of the mobility spectrum has been used in the smoothing and decreasing procedures. Using the extended iteration procedure, one can obtain good resolutions for the mobility spectrum, and the mobility spectrum is not sensitive to the form of the first trial function. The extended procedure eliminates 
a whole class of solutions. However, some further modifications of the resulting mobility spectra are possible using more specified requirements following from the "physical sense" of the mobility spectrum.

\section{Acknowledgments}

The author is grateful to Professor R.R. Gałazka, Dr. M. Górska, Dr. A. Eusakowski and to Dr. J. Wróbel for numerous helpful discussions.

\section{References}

[1] J.W. McClure, Phys. Rev. 101, 1642 (1956).

[2] J. Kołodziejczak, Acta Phys. Pol. 20, 379 (1961).

[3] W.A. Beck, J.R. Anderson, J. Appl. Phys. 62, 541 (1987).

[4] Z. Dziuba, M. Górska, J. Phys. III (France), to be published.

[5] R.E. Bellman, R.E. Kalaba, J. Lockett, Numerical Inversion of the Laplace Transform. Modern Analytical and Computational Methods in Sciences and Mathematics, Vol 4, American Elsevier, New York 1966.

[6] Z. Dziuba, Phys. Status Solidi B 140, 213 (1987).

[7] Z. Dziuba, R. Kowalczyk, Phys. Status Solidi B 119, K11 (1983).

[8] I.M. Tsidilkovskii, Yu.G. Arapov, M.L. Zvareva, N.B. Brandt, V.A. Kulbachinskii, I.N. Gorbatyuk, A.I. Elizarov, Phys. Status Solidi B 148, 197 (1988).

[9] I.M. Tsidilkovskii, Yu.G. Arapov, M.L. Zvareva, in: 19th Inter. Conf. Phys. Semicond., Ed. W. Zawadzki, Institute of Physics of Polish Academy of Sciences, Vol. 2, Warsaw 1988, p. 1209. 